\title{
Appropriate Resource Allocation for Effective Mobile Communication in 4G LTE- Advanced
}

\author{
J. Rejina Parvin and S. Karthika \\ Department of Electronics and Communication Engineering, Sri Krishna College of Engineering and Technology, \\ Coimbatore-641008,Tamil Nadu, India; rejinaparvinj@skcet.ac.in, karthikas@skcet.ac.in
}

\begin{abstract}
Objective: Neighboring cells become progressively worse especially for cell edge users due to Inter Cell Interference (ICI) in Orthogonal Frequency Division Multiple Access (OFDMA). Frequency Reuse Factor is to mitigate ICI by proper allocation of resources to users in network layout. Methods/Statistical Analysis: This study presents a self-organized resource allocation scheme for automatically selecting the reuse factor based on number of cell edge users and cell center user. Findings: This study explains about the new scheduling metric based on transmitted energy per bit and this provides the resources to users which are having low ration of transmitted energy per bit to avoid the wastage of resources. Applications/Improvements: Due to consideration of different reuse factor and also scheduling metric, this algorithm provides better performance for edge users in terms of Energy cell efficiency, Signal to Noise Interference Cell Ratio (SINR) and Fairness Index.
\end{abstract}

Keywords: Center Users, Edge Users, 4G LTE Advanced, Round Robin (RR), Maximum Carrier to Interference Ratio (MCI)

\section{Introduction}

Cellular network concept is one of the important concepts in wireless communication. The system operates by splitting the coverage area into various cells or zones in which each cell has its own resource. The cell is of hexagonal shape. The signals transmitted from Base Station to the mobile equipment are termed as downlink/forward link. The prime motive of the cellular communication is proving service to the maximum number of users by using the concept of reusing the available resources. Each Base Station is connected to the Mobile Switching Center (MSC) in which Public Telephone System (PTS) acts as a backbone of it. As there is various numbers of users for transmitting the data, multiple schemes can be used to share the available bandwidth. In the cellular system, the network service provided area into various subareas and is called as cells. In each cell, different user has to use only the available bandwidth but each with different carrier frequency $(\mathrm{fc})$. Where as in the neighboring cell, each user uses different frequency in order to minimize the co-channel interference. Since the number of users keep on increasing, it is difficult to provide service efficiently with the available frequency. At such cases, the cells will be allocated by the same frequency which is located far away. Here the probability of interference will be lesser as the signal strength gets decreases with increase in the distance. In three ways, the channel capacity can be improved in the cellular systems.

Reuse of the same frequency, multiple access scheme and spectrally efficient modulation can be adopted to increase the channel capacity. These also results in less interference between the channels. Various research works have been carried out towards resource allocation in cellular systems. Allocation based on the priority is one of the prime ides behind resource allocation. In addition 
to that Self Organized Resource Allocation (SORA) is one of the methods which allocates the resource in a dynamic fashion. This study gives the overall idea of resource allocation in cellular network architecture.

The study is organized as follows: section 1 gave a brief introduction about the cellular network and its limitations. Section 2 and 3 describes about the existing works and proposed system model respectively. Proposed algorithm and Results and discussion is given in section 4 and 5 respectively. In section 6 , the work is concluded with its merits, limitations and future enhancement.

\section{Literature Survey of Existing Methods}

In OFDMA, the frequency selective wideband channel is divided into number of non-frequency parallel orthogonal narrowband subcarriers. The interference from neighbouring cells is considered as major challenge that degrades the performance of OFDMA cellular systems particularly for edge users. The concept of mitigates ICI by allocating the orthogonal resources to the overlapping cells. In this, cancellation of ICI is done by frequency reuse and power control techniques. The effective reuse factor of the available frequency resources can highly improve the system capacity. With small reuse factor, more resources are allotted for each cell. Low reuse factor results heavy ICI especially for edge users and degrades the system performance in terms of coverage and capacity.

The resources are allocating to the outer UEs in an efficient manner by use of direct combination between adjacent BSs to handle the interference. The conventional method is to increase the cochannel distance using high cluster size which can mitigate ICI efficiently. Increasing the cluster size which causes the decrease of available frequency reuse resources for each cell which results in restricted data transmission rate and lower the system spectral efficiency. Several methods are there to reduce ICI such as ICI Cancellation (ICIC) and ICI Avoidance/ Coordination etc., is elucidated ${ }^{1}$ and shown in Figure 1. In ICIC methods, interference reduction is done by applying restrictions to resource management in coordinated manner. Resource coordination is done by time, power or frequency. A theoretical concept of reuse partitioning methods that allocating different reuse factors to the users to mitigates ICI2. FFR is introduced by $3 \mathrm{GPP}$ cellular

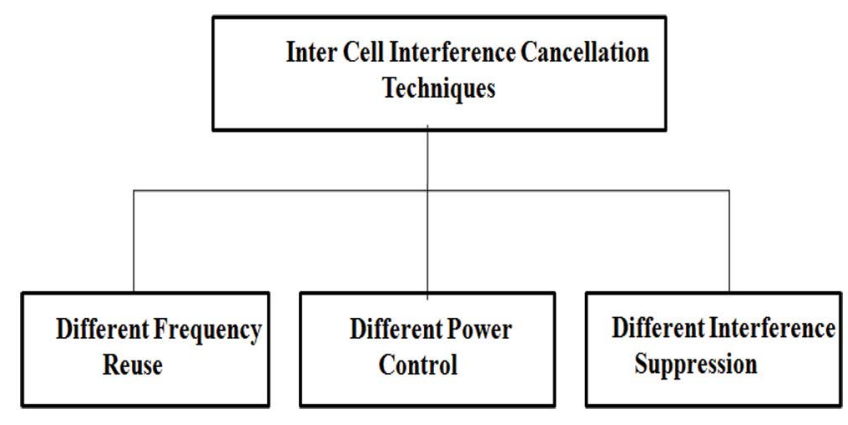

Figure 1. ICIC Techniques.

systems and discussed various methods which are used to reduce ICI3. FFR effectively mitigates interference by applying different frequency reuse factors to users based on their regions. The concept of FFR is to split the total bandwidth into two frequency bands. Those divided frequency bands are given to two regions i.e. inner and outer regions. The concept of FFR is to divide the total bandwidth into two regions i.e. Cell Center Area (CCA) and Cell Edge Areas (CEA). The UEs which are locating in CCA are Cell Center Users (CCU) and UEs are located in CEA are referred as Cell Edge Users (CEU). Low reuse factor is assigned to CCU which having high signal quality and high reuse factor is assigned to CEU due to low signal strength. CCU use low reuse factor to achieve high spectral efficiency

The UE in inner regions with best signal quality can use low frequency reuse factor where UE in outer regions uses high reuse factor compared to inner regions. A system has been developed a method to improve the capacity in FFR4. A method for user's differentiation as inner users and outer users based on geographical locations then applying FFR schemes for scheduling ${ }^{2-t}$. The optimization of design parameter for FFR using graph theory and optimization methods are explained ${ }^{4-6}$. The author ${ }^{4}$ explained that reuse factor- 3 is provides better performance for edge users i.e. users are located in outer regions. By using this choice, the capacity gain upto $25 \%$ in OFDMA systems ${ }^{7}$. It is proposed ${ }^{8}$ and optimization technique for user's differentiation. It was elucidated that ${ }^{9}$ a method that selects the optimal region size for inner and outer users for each cell and it allocates optimal frequency reuse methods for different regions to maximize the system throughput based on user satisfaction metric. The major limitations of FFR are resources are underutilization in the cell edge region since high reuse factor is employed. The experimental solution at the desired temperature. The accuracy in the temperature measurement is $\pm 0.01 \mathrm{~K}$. Generalized 
Fractional Frequency Reuse (GFFR) proposed which is used for irregular cell structure and this scheme adapts the utilization of resources based on the level of interference for the cell edge users and this method used to select the partition of the frequency bands based on user satisfaction metric ${ }^{10}$. The enhanced ICIC (eICIC) is introduced for the improvement of coverage and capacity in multilayer LTE network ${ }^{11}$.

The variant of frequency reuse is mentioned $\frac{12}{}$ where the power transmission for edge users is amplified than the center users. A method has been proposed scheduling method based on Soft Frequency Reuse (SFR) $\frac{13}{}$ and this method classify the users in an efficient manner and improves the fairness and throughput among the multi users. In ${ }^{14}$ there proposed an adaptive method where multiple BSs jointly select the joint transmission strategies based on location of UEs and it requires lower feedback rate. The different types of ICIC methods are surveyed ${ }^{15}$. A novel ICIC method ${ }^{16}$ by allotting the different reuse factor values based on user positions and it is explained. The different frequency reuse factors are allotted to the UEs based on regions of cells. The cell regions are segmented into many regions and different bands are allotted to the $\mathrm{UEs}^{1 \frac{17}{}}$.

\section{Proposed System Model}

In cellular network, it is necessary to understand the need for resource reusing. The main objective of the mobile systems was to place a high-powered antenna on a tall building or mountain. Even though, this approach offered a wide coverage area. It was almost impossible to reuse the resource without creating the interference to the other users. In the year of 1970, Bell Laboratory supports low number of calls. Later, this fact was changed due to wireless cellular communication. Cells may have different radii in the range from $1 \mathrm{~km}$ to $30 \mathrm{~km}$, the boundaries of them can overlap between neighbouring cells and also large cells can be partitioned into smaller cells. The emerging technology in wireless communication brings critical setbacks to the network. Both users and service providers desire cutting edge performance from their devices and technologies. On the one hand, major licensed bands are allocated to the television broadcasting and military applications. On the other hand, unlicensed bands are occupied by the overcrowded devices such as cordless phone, Wireless Local Area Networks (WLAN) and home entertainment purposes. Thus, reusing the limited resource within the designated band is done to overcome the scarcity issues.

The network model is considered for the research work is downlink OFDMA cellular systems. The network layout consists of 19 eNBs. The reference cell is 0 for which interference will be considered from other eNB transmitting in the same Time Transmission Interval (TTI) as eNB in the reference cell as shown in the Figure 2. $U$ is the number of users placed randomly in network layout. Each cell consists of one eNB.

The propagation model (Huq et al.) is considered in this model as follows:

Line of Sight (LOS):

PL $(d B)=22 \log (d)+34.02 ; 10 m<d<300 m$

PL $(\mathrm{dB})=40 \log (\mathrm{d})-11.02 ; 300 \mathrm{~m}<\mathrm{d}<5 \mathrm{~km}$

Non - Line of Sight (NLOS):

PL $(\mathrm{dB})=39 \log (\mathrm{d} / 1000)+136.8245$

In 3GPP LTE Standard, the available frequency spectrum is divided into $\mathrm{m}$ resource blocks. Each Resource Blocks (RBs) consists of 12 subcarriers of total bandwidth of $180 \mathrm{kHz}$. Scheduling is performed for every TTI in order to allocate the RBs to the users. The interference within the reference cell to the users coming from all other eNB transmitting in the same TTI as follows:

$$
\Gamma_{c, u}^{m}=\frac{P_{c, u}^{m}\left|h_{c, u}^{m} W_{c, u}^{m}\right|^{2}}{\sum_{u^{\prime} \neq u}^{U} P_{c, u^{\prime}}^{m}\left|h_{c, u}^{m} W_{c, u^{\prime}}^{m}\right|^{2}+\left|\eta_{c, u}^{m}\right|^{2}}
$$

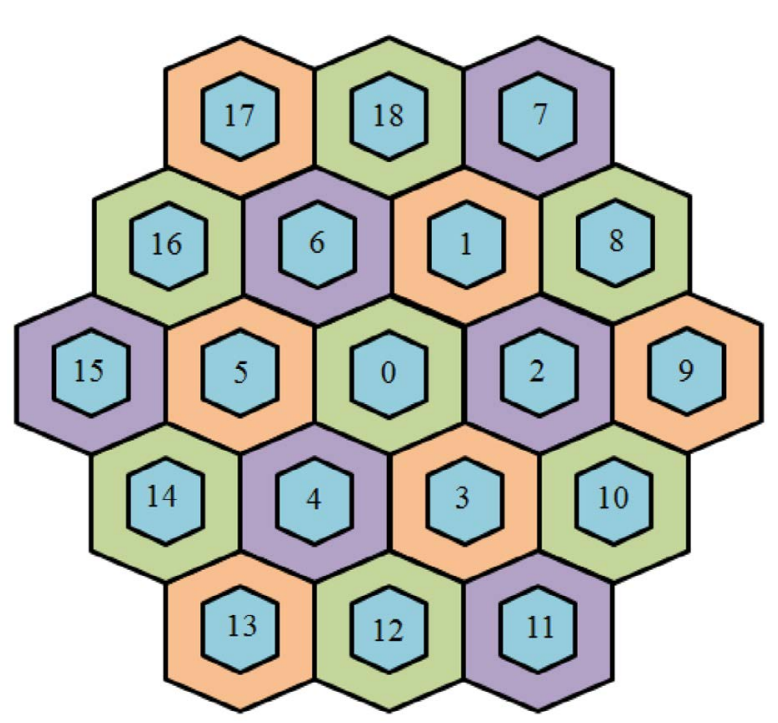

Figure 2. System Model with 19 eNBs. 
Where is the total transmitted power of uth user of $m$ PRB in c cell, is the Additive White Gaussian Noise (AWGN) received at UE, is the complex channel vector combines of path loss, shadowing, fast fading and link between UE and eNB.

Using the Shannon theorem, the throughput of uth $\mathrm{UE}$ on mth RB is given by,

$$
T_{c, u}^{m}=\Delta f^{\star} \log _{2}\left(1+\frac{\Gamma_{c, u}^{m}}{\varepsilon}\right)
$$

Where is the available bandwidth allocated to the uth user and $\varepsilon$ is a constant related to the target Bit Error Rate (BER) and it is given by,

$$
\varepsilon=\frac{-\ln (5 B E R)}{1.5}
$$

The total throughput of uth UE in the serving cell is given by,

$$
T_{c, u}=\sum_{m} \mu_{u, m}{ }^{\star} T_{c, m}^{m}
$$

Where $\mu \mathrm{u}, \mathrm{m}$ indicates that assignment of $\mathrm{mth}$ resources to the user in the cth cell and it is given as follows:

$$
\mu_{u, m}=\left\{\begin{array}{lc}
1, & \text { mth resource is assigned to the } u^{\text {th }} \text { user } \\
0 & \text { otherwise }
\end{array}\right.
$$

\subsection{Self Organized Resource Allocation Algorithm}

The main characteristic of frequency reuse in cellular systems is to increase the capacity and coverage. The main idea of frequency reuse is to divide the frequency bands into many narrow bands. Each narrow band is assigned to a cell. Reuse partitioning is the interference mitigation techniques by applying different reuse factors to the different regions in cell.

The available frequency spectrum is reused in each cell is Frequency Reuse -1 (FR-1) ${ }^{18-19}$. FR-1 results worst channel interference scenario. In Frequency Reuse -3 (FR-3) $)^{\frac{18}{}}$, the available frequency spectrum is divided into three equal sub bands; each sub band is allocated orthogonally to the adjacent cells. FR-3 is better when compared to FR -1 in terms of co channel interference. The FR- 1 and FR-3 is given in Figure 3 a-b respectively. The spectrum is divided into two groups i.e. inner and outer groups. FR-1 is allotted to Cell Center Users (CCU) (i.e. users are in inner regions) and FR-3 is allotted to the
Cell Edge Users (CEU) (i.e. users are in outer regions) and it is given in Figure 3. The three different bands are allocated to the users in outer and inner regions. The users are differentiated as CCU and CEU by SINR values as given in the Equation 3. The available spectrum $\beta$ is divided into $\beta$ FR- 1 and $\beta$ FR-3. In proposed algorithm, outer bands are partitioned into three bands to avoid interference and it is shown in Figure 4. The inner band $\beta F R-1$ is used with FR- 1 and $3^{*} \beta$ FR-3 is used with FR-3.

$\beta \mathrm{FS}=\beta \mathrm{FR}-1+\beta \mathrm{FR}-3$

Where $\beta F S$ is the available spectrum for each cell and $\beta F R F$ is the effective frequency reuse factor. Each BS distributes the equal power over the available bands of its cell in downlink data transmission.

The users are randomly populated in the network layout. The optimal resource allocation between the

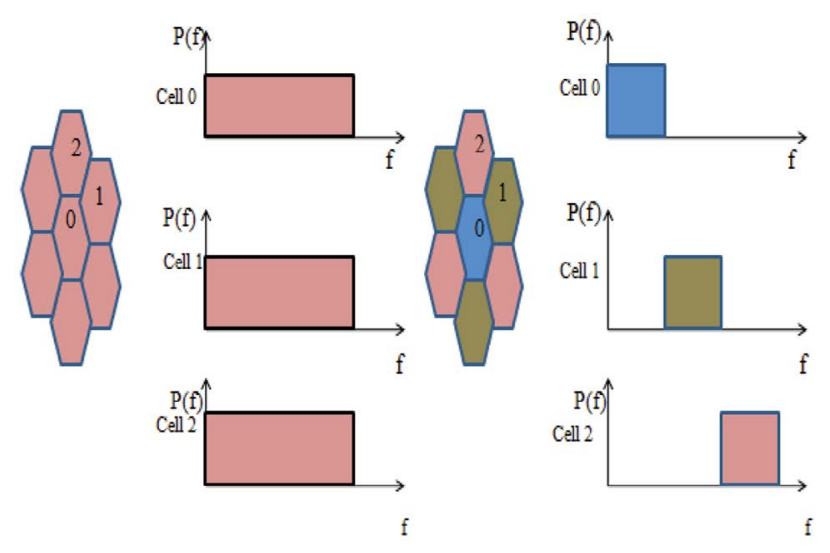

Figure 3. a) Frequency Reuse - 1 b) Frequency Reuse -3.

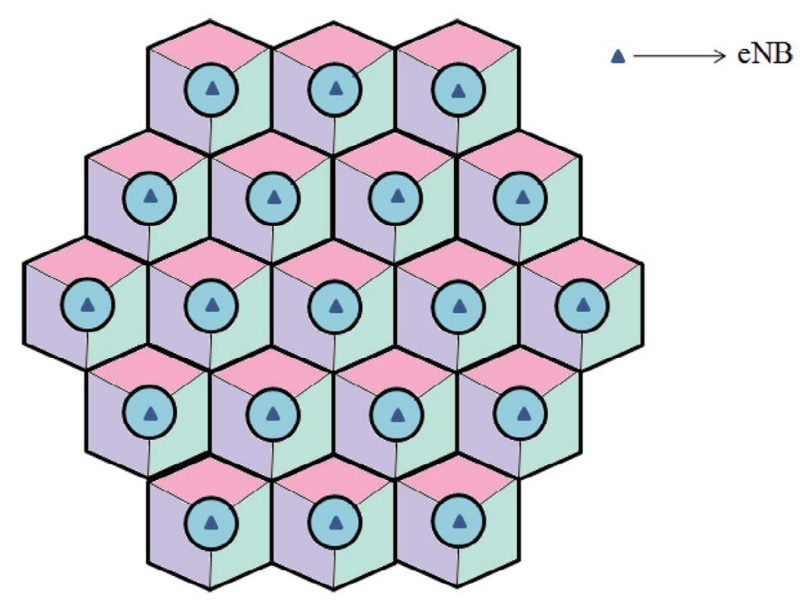

Figure 4. Pictorial representation of Scenario Proposed Algorithm. 
regions is to improve the performance of cell edge users. The user's differentiation can be based on SINR measurements at UE. If received SINR is greater than the threshold SINR then the user is located in inner regions i.e. center users else it is edge users. Threshold SINR is assumed to be $3 \mathrm{~dB}$. In this proposed method, SORA automatically selects the different reuse factors based on the location of users. Joint transmission is used to increase the signal strength of edge users. The resource allocation to users based on coordination between neighboring NB through a message passing approach using X2 interface. The central serving eNB collects the information and distribute the resources to the other eNB in network. In this scheme, each UE measures the downlink SINR on each RB based on the reference signals received from the serving eNB in each TTI and it sends the SINR measurements as feedback to the serving eNB through Channel Quality Indicator (CQI) measurements. The users are classified as CCU and CEU based on SINR measurements. Frequency Allocation Variable (FAV) is introduced for resource allocation and it will allocate the resource depends on number of users in serving cell. Each eNB calculates the number of center users and edge users in a cell. Based on number of users in serving cell, FAV will allocates the resources to the active users and is given by,

$$
\begin{gathered}
F A V=\frac{\text { Number of Edge Users }}{\text { Number of Center Users }} \\
=\frac{\beta_{F R-3}}{\beta_{F R-1}}
\end{gathered}
$$

Where is the reuse factor for edge users? Based on the Equation (10), the reuse factor for CCU is given by,

$$
\beta_{F R-1}=\frac{\beta}{3^{\star} F A V+1}
$$

The reuse factor for edge users is given by,

$$
\beta_{F R-3}=\frac{\beta-\beta_{F R-1}}{3}
$$

The serving eNB will sends the FAV to the nearby cell through coordination for optimal resource allocation. The flowchart of the resource allocation is given in the Figure 5. The steps of the resource allocation are discussed as follows:

Step 1: The number of users, number of subcarriers, number of eNBs, Transmission Power of eNB and $\mathrm{UE}$ and $\mathrm{TTI}=1 \mathrm{~ms}$ are initialized.

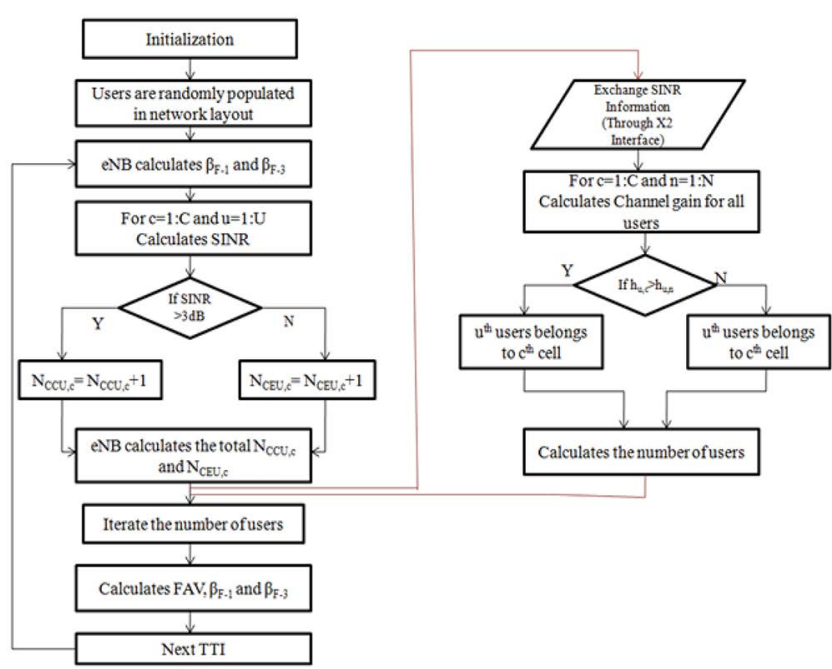

Figure 5. Flowchart of SORA Algorithm.

Step 2: The users are placed randomly in the network layout.

Step 3: The serving eNB allocates the resources to the users.

Step 4: Users are differentiated as CCU and CEU based on SINR measurements.

Step 5: If downlink SINR for UE is greater than the SINR threshold then the user is CCU else CEU.

Step 6: Each serving eNB calculates the number of CCU and CEU.

Step 7: FAV value is calculated by Equation. Based on FAV, resources are allocated to the users.

Step 8: eNB coordinated through X2 interface to verify the number of edge users. Because edge users can be move to nearby cells.

Step 9: The number of users are iterated for every TTI.

Step 10: Then the data transmission are processed.

\section{Proposed Algorithm}

The basic flowchart of Energy efficient scheduling algorithm is given in Figure 5. Users are differentiated into Center Users (CU) and Edge Users (EU) based on Signal to Interference Noise Ratio (SINR) and is explained ${ }^{20}$. After the process of differentiation, resource blocks are allocated among users and it reduces interference. In this study, new criterion energy per bit is taken for energy efficiency by proper allocation of resources. $\mathrm{RR}$ and MCI algorithm based on channel throughput. More resources are allocated to users which causes more energy due to increase of subscribers. Users has poor 
channel conditions cannot utilize the full radio resources. To put this end, scheduling metric is considered and it is the ratio of energy per bit. As a result of this algorithm, the network with low transmit energy is highly effective to assign the resources. This scheduling metric is used to assign the priorities among users from low to high.

\section{Calculation of Scheduling Metric}

In this resource allocation scheme, the scheduling metric chooses the users based on the ratio of transmitted energy to the number of transmission bits on resources $b$ for users $n$. Users to be assigned in an order from lower scheduling metric. Scheduling metric is given by,

$$
\text { Scheduling metric }=\operatorname{argmin} \frac{\xi\left(B_{n}^{b}\right)^{\star} t}{h_{n}^{b} B_{n}^{b}}
$$

Where scheduling metric denotes the index of the selected users and resources respectively, is the channel gain, is the number of transmission bits and is the minimum transmitted power required for the number of transmission bits and it is given by,

$$
\xi\left(B_{n}^{b}\right)=\frac{\left(\sigma_{n}^{b}\right)^{2}}{3}\left[Q^{-1}\left(\frac{B E R}{4}\right)\right]^{2}\left(2^{B_{n}^{b}}-1\right)
$$

Where is the noise variance? Scheduling metric depends on the minimum transmitted power for the number of transmission bits and it also shown the effects of bits changes in the transmission bits.

Let is the maximum transmitted power by the BSs to the $\mathrm{n}^{\text {th }} \mathrm{UE}$ on $\mathrm{b}^{\text {th }}$ resources blocks. Eventually, the scheduling metric can be expressed as,

$$
\text { Scheduling metric }=\operatorname{argmax}\left\{\frac{C_{n}^{b} B_{n}^{b}}{R_{n}^{b}}\right\}
$$

Where Excess Channel gain

$$
C_{n}^{b}=\frac{1}{h_{n}^{b}\left(B_{n}^{b}\right)}-\frac{1}{h\left(B_{n}^{b}\right)_{\min }}
$$

Received energy per bit is given by,

$$
R_{n}^{b}=\xi\left(B_{n}^{b}\right) T
$$

The scheduler allocates the resources to the users which are having low scheduling metric and also having high channel gain. At the condition of users having same channel gain, then the resources are allocated to users are having lowest received energy per bit.

\section{Results and Discussions}

The comparison of the proposed scheduling algorithm is explained in following section.

\section{Performance Metrics}

Energy Efficiency Metric is referred as successfully transmitted bits over the transmission power and it is measured in bits/Joule. It is given by,

$$
\zeta=\frac{\text { Throughput }}{P_{T}}
$$

Where $\mathrm{P}_{\mathrm{T}}$ is the transmission power.

\section{Fairness Index}

To measure the equivalence of the resources. It is given by,

$$
F I=\frac{\sum_{i=1}^{n} \sum_{j=1}^{n}\left|x_{i}-x_{j}\right|}{2 n^{2} \bar{x}}
$$

Where $\mathrm{x}$ is an observed EE value, $\mathrm{n}$ is the total number of observed value and is the mean value. It lies between 0 and 1 . If the value is 1 for complete fairness otherwise its equals to 0 . The simulation parameters are given in Table 1. The proposed scheduling algorithm is compared with other schedulers such as Round Robin (RR), Maximum Carrier to Interference ratio (MCI) and Proportional Fairness $(\mathrm{PF})^{21-22}$. The simulations are done in central single cell and remaining cells are considered as interferers. Users are placed randomly at various locations. The antenna configuration is considered for simulations are $2 \times 2$. The minimum distance is considered between UE and eNB is $30 \mathrm{~m}$ and Noise spectral density is assumed as $-174 \mathrm{dBm}$. The different performance measures like Average Energy Efficiency for edge and center users, Capacity and SINR are evaluated. The resource blocks are simulated based on channel bandwidth in each TTI.

Figure 6 shows the flow chart which describes the algorithm in the following steps:

Step 1: Initialize the number of Base stations (eNBs), number of User Equipment (UEs) and Channel Bandwidth

Step 2: Place the users randomly in network layout.

Step 3: eNB allocate $\mathrm{B}_{\mathrm{FR}-1}$ and $\mathrm{B}_{\mathrm{FR} 3}$ (Frequency Reuse -1 and Frequency Reuse -3 ) to center and edge users respectively. 
Table 1. Simulation Parameters

\begin{tabular}{ll}
\hline Parameters & Value \\
\hline Bandwidth & $20 \mathrm{MHz}$ \\
eNB power & $46 \mathrm{dBm}$ \\
UE antenna gain & $11 \mathrm{dBi}$ \\
Carrier Frequency & $2.6 \mathrm{GHz}$ \\
Number of Resource Blocks & $100 \mathrm{RBs}$ per slot \\
$1 \mathrm{RB}$ & $12 \mathrm{subcarriers}$ \\
$1 \mathrm{RB}$ & $180 \mathrm{kHz}$ \\
Subcarrier Spacing & $15 \mathrm{kHz}$ \\
Number of OFDM symbols & $7 \mathrm{symbols}$ \\
Number of subcarriers & 1200 \\
Channel Model & LTE Channel model \\
Modulation scheme & $16 \mathrm{QAM}$ \\
Inter site Distance & $500 \mathrm{~m}$ \\
\hline
\end{tabular}
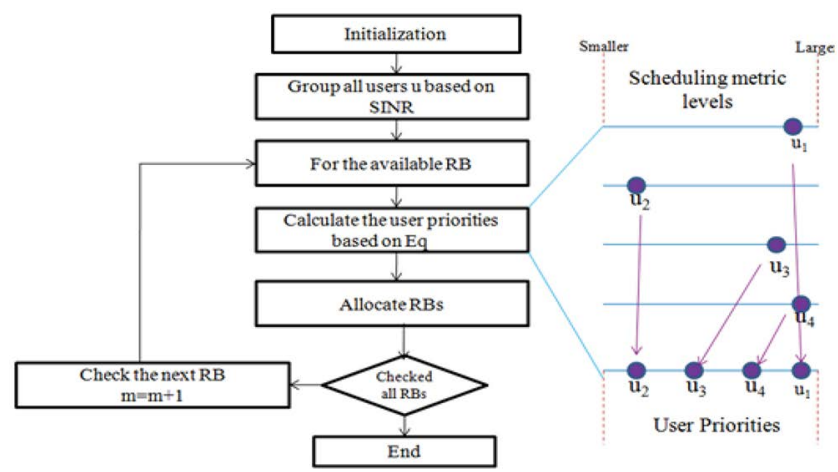

Figure 6. Flowchart of the Proposed Algorithm.

Step 4: Calculate SINR for all link between Base station and Users. If SINR value for user $<3 \mathrm{~dB}$, then the user is in edge, else the user is in center.

Step 5: Count the total number of edge and center users. eNBs sends an information about the total number of edge and center users to the Central eNB.

Step 6: After allocating the resources to cell, the transmission is done based on user priorities.

Step 7: Calculate the user priorities based on transmit energy and number of transmission bits. User are allocated fromlower to higher ratio of transmit energy over the transmission bits.

Step 8: Allocate the resources to users based on priorities.

Step 9: Check the Remaining Resource block for allocation.

Step 10: Stop the program.
The parameters such as number of nodes transmit power, UE antenna power and antenna gain is initialized. eNB allocates all resources to the serving UEs for first TTI. Users are differentiated into inner users and outer users by SINR measurements at UE side. Then resource blocks are grouped into two groups' i.e. inner and outer groups. From the available of inner and outer groups of resource blocks, resource blocks are allocated to the users based on user priorities. The remaining resources are to be checked for the next transmission. From Figure 7, it is observed that CDF SINR comparison for different frequency reuse. In FR Technique, reuse- 3 is used for resource allocation. From among those, proposed scheme with SORA algorithm $^{20}$ provides better performance in terms of SINR. SORA algorithm provides better SINR. The resources are allocated to twodifferent groups of users i.e. CU or EU based on channel characteristics. Before assigning priorities to users, available subcarriers are grouped into two group's i.e. inner and outer groups. From the available of subcarriers of two groups, priorities are allotted to the users based on excess channel gain. From the Figure 7, SORA provides better SINR for the TTI.

Figure 8 shows the CDF capacity comparison of proposed schedulers with other increases the overall user's capacity. Comparison of Average Cell Energy Efficiency (AEE) for users and Edge users are given in Figures 9-10 respectively. It shows that EE Comparison for the maximum of 500 UEs in each cell. For example, when the users are 100, the algorithm shows $35 \%$ improvement than

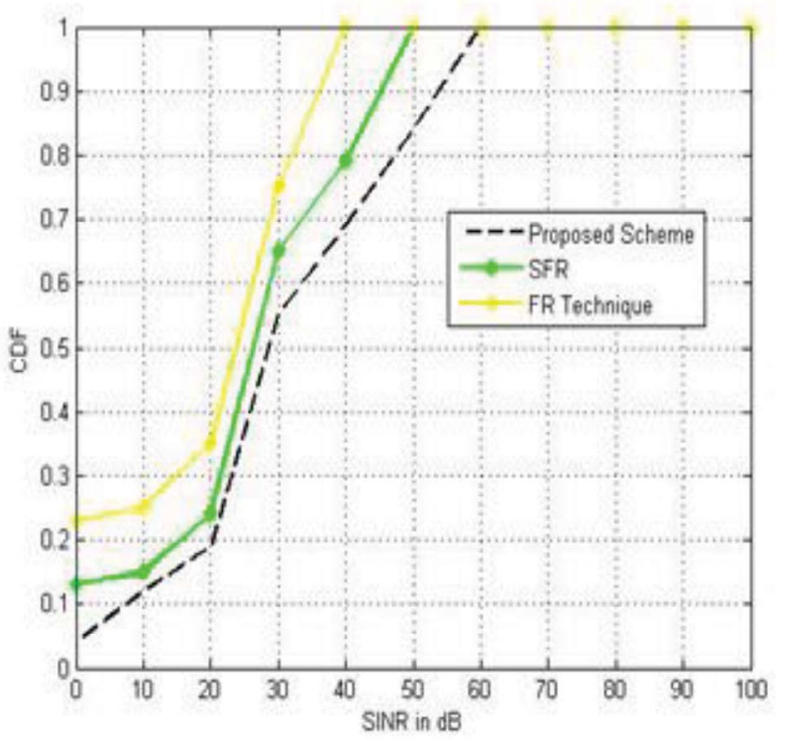

Figure 7. Cumulative Distribution Function of Figure. 
the MCI due to increasing energy per bit. Transmitted energy per bit becomes more critical because energy is a limited resource. To overcome this, new criterion that ratio of transmit energy per number of transmitted bits is considered in scheduling algorithm. In MCI, resources are allocated to the users based on the maximum channel gain so MCI provides low AEE when compared to the proposed scheduling algorithm. RR provides low AEE

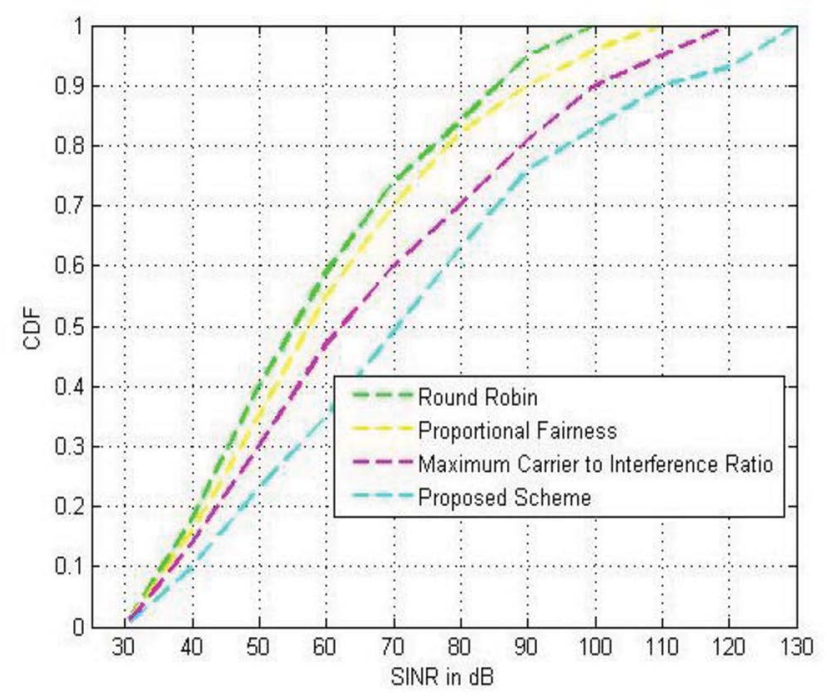

Figure 8. Cumulative Distribution SINR for various schemes with other schedulers Function of SINR for proposed method.

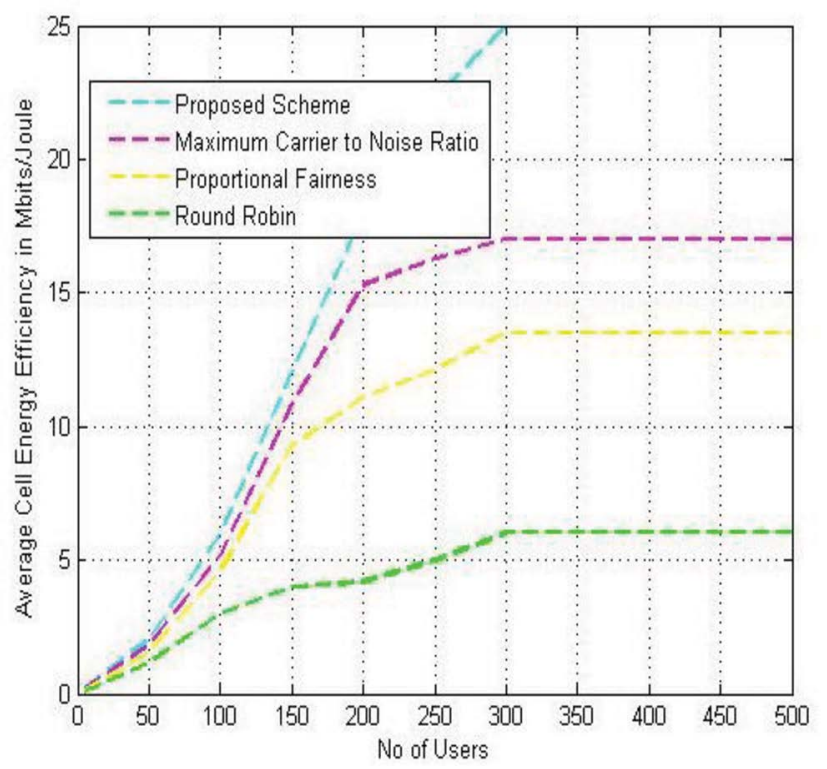

Figure 9. Comparison of Average Cell EE for all users in scenario. due to allocate more resources to the users eventhough UEs has poor channel. Figure 10 shows the comparison of AEE for edge users with the maximum of 500 users. In a scenario, 150 users are considered as edge users i.e. users which are having SINR lower than the $3 \mathrm{~dB}$ and remaining are considered as center users. When the users are at maximum of 100 users, proposed scheduling algorithm achieves improvement of $13 \%$ compared to MCI. MCI provides low EE for edge users due to low channel gain for edge users because it provides resources to the users having high channel gain. Due to the low signal gain for edge users, sufficient resources are not allocated. Fairness Index comparison of users at various locations is shown in Figure 11. Proposed scheme improves fairness for the

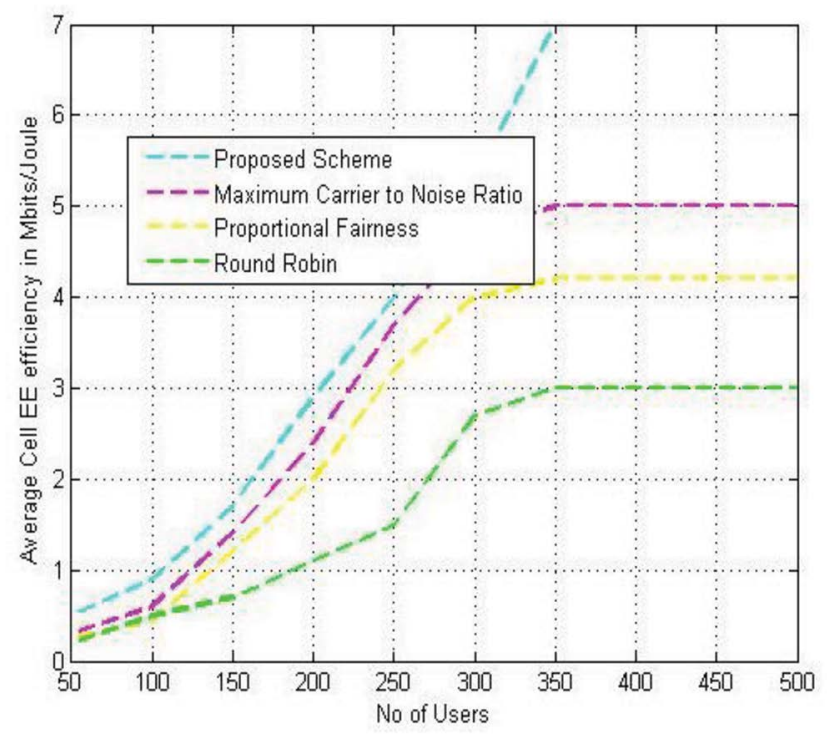

Figure 10. Comparison of Average Cell EE for Edge users.

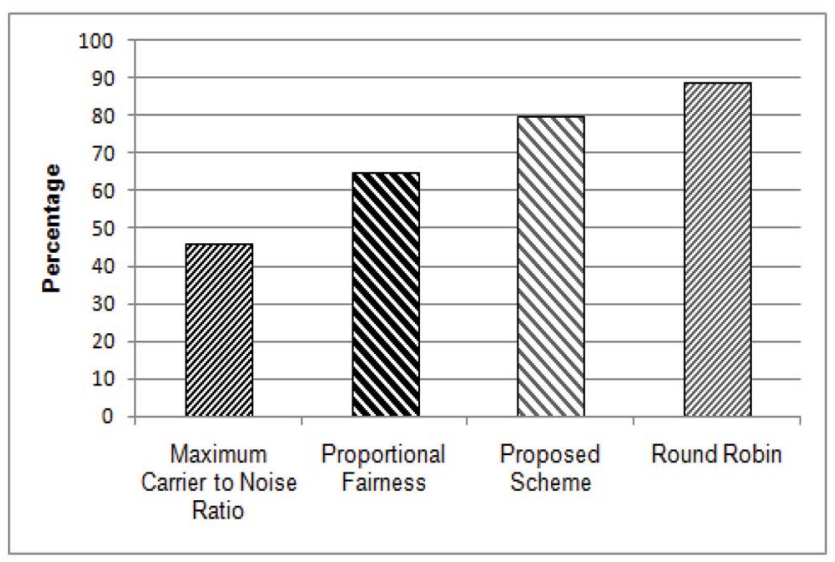

Figure 11. Comparison of Fairness Index. 
various numbers of users. MCI provides low fairness to the users due to allocation of resources based on the best channel gain. In MCI, the users located near to eNB will receive resources but edge users are not able to receive resources due to low signal strength and this condition not allowing the appropriate allocation of resources to all users in serving cell.

\section{Conclusion}

This study explains about Energy Effective scheduling algorithm. From the above analysis, existing schedulers such as RR, MCI and PF provides resources to the center users not to edge users which are having low signal strength. This algorithm provides resources to all users. The system level simulations suggest that proposed method provides better energy efficiency for users in serving cell. In this interference is avoided by allocating of particular resources to $\mathrm{CU}$ and EU, then the resources are allocated based scheduling metric which is ratio of transmitted energy per bit to the number of transmitted bits.

\section{References}

1. Pateromichelakis E, Shariat M, Ul Quddus A, Tafazolli R. On the evolution of multi-cell scheduling in 3GPP LTE/LTE-A. IEEE Communications Surveys \& Tutorials. 2013; 15(2):701-17. https://doi.org/10.1109/ SURV.2012.071812.00127

2. Fujii $\mathrm{H}$, Yoshino $\mathrm{H}$. Theoretical capacity and outage rate of OFDMA cellular system with fractional frequency reuse. IEEE Vehicular Technology Conference. 2008; p. 1676-80.

3. Saquib N, Hossain E, Kim DI. Fractional frequency reuse for interference management in LTE-advanced hetnets. IEEE Wireless Communications. 2013; 20(2):113-22. https://doi.org/10.1109/MWC.2013.6507402

4. Assaad M. Optimal fractional frequency reuse (FFR) in multi cellular OFDMA system. IEEE Vehicular Technology Conference. 2008; p. 1-5.

5. Chang RY, Tao Z, Zhang J, Kuo CC. A graph approach to dynamic fractional frequency reuse (FFR) in multi-cell OFDMA networks. IEEE International Conference on Communications. 2009; p. 1-6.
6. Chang RY, Tao Z, Zhang J, Kuo CC. Multicell OFDMA downlink resource allocation using a graphic framework. IEEE Transactions on Vehicular Technology. 2009; 58(7):3494507. https://doi.org/10.1109/TVT.2009.2014384

7. Lei H, Zhang L, Zhang X, Yang D. A novel multi-cell OFDMA system structure using fractional frequency reuse. IEEE 18th International Symposium on Personal, Indoor and Mobile Radio Communications. 2007; p. 1-5.

8. Amer M. Optimal configuration of fractional frequency reuse system for LTE cellular networks. IEEE Vehicular Technology Conference. 2012; p. 1-5. PMid:23031385

9. Bilios D, Bouras C, Kokkinos V, Papazois A, Tseliou G. Optimization of fractional frequency reuse in long term evolution networks. Wireless Communications and Networking Conference. 2012; p. 1853-7.

10. Chen L, Yuan D. Generalizing and optimizing fractional frequency reuse in broadband cellular radio access networks. EURASIP Journal on Wireless Communications and Networking. 2012; 230:1-15.

11. Pedersen KI, Wang Y, Strzyz S, Frederiksen F. Enhanced inter-cell interference coordination in co-channel multi-layer LTE advanced networks. IEEE Wireless Communications. 2013; 20(3):120-7. https://doi. org/10.1109/MWC.2013.6549291

12. Xiang Y, Luo J, Hartmann C. Inter-cell interference mitigation through flexible resource reuse in OFDMA based communication networks. European Wireless. 2007; p. 1-7.

13. Lee D, Li GY, Tang S. Intercell interference coordination for LTE systems. IEEE Transactions on Vehicular Technology. 2013; 62(9):4408-20. https://doi.org/10.1109/ TVT.2013.2263814

14. Zhang J, Andrews JG. Adaptive spatial intercell interference cancellation in multicell wireless networks. IEEE Journal on Selected Areas in Communications. 2010; 28(9):145568. https://doi.org/10.1109/JSAC.2010.101207

15. Hamza AS, Khalifa SS, Hamza HS, Elsayed K. A survey on inter-cell interference coordination techniques in OFDMAbased cellular networks. IEEE Communications Surveys \& Tutorials. 2013; 15(4):1642-70. https://doi.org/10.1109/ SURV.2013.013013.00028

16. Xiao D, Yu X, Yang D. A novel downlink ICIC method based on user position in LTE-advanced systems. IEEE Vehicular Technology Conference (VTC Fall). 2012; p. 1-5.

17. Huang J, Xiao P, Jing X. A downlink ICIC method based on region in the LTE-Advanced system. IEEE 21st International Symposium on Personal, Indoor and Mobile Radio Communications Workshops. 2010; p. 420-3. 
18. Halpern SW. Reuse Partioning in Cellular Systems. IEEE Vehicular Technology Conference. 1983; p. 322-7.

19. Sternad M, Ottosson T, Ahlen A, Svensson A. Attaining both coverage and high spectral efficiency with adaptive OFDM downlinks. IEEE Vehicular Technology Conference. 2003; 4:2486-90.

20. Karthika SP, Indumathi P, Rajakumar R. Performance analysis of frequency reuse in cellular networks in fading channels. Journal Wireless Communications \& Mobile Computing. 2016; 16(7):850-67.

21. Kwon $H$, Birdsall T. Channel capacity in bits per joule. IEEE Journal of Oceanic Engineering. 1986; 11(1):97-9. https:// doi.org/10.1109/JOE.1986.1145138

22. Marshall AW, Olkin I, Arnold BC. Inequalities: theory of majorization and its applications. Springer Series in Statistics. 1979; p. 1-940. 